\title{
LCF and crack growth: recent results obtained by DIC
}

\author{
Luca Patriarca, Stefano Foletti, and Stefano Beretta* \\ Politecnico di Milano, Department of Mechanical Engineering, via La Masa 1, 20156, Milano
}

\begin{abstract}
Nowadays, several components for aerospace and energy applications (i.e., turbine disks, offshore pipelines, and combustor chambers) are designed following a safe-life approach in the low cycle fatigue (LCF) regime. As also in the LCF regime it is worth adopting a damage tolerance design, fatigue life is assessed considering a crack propagating from the first load cycle, starting from an initial defect present in the component's most-stressed region or from a microstructural feature. In this frame, fatigue crack growth is described considering LCF conditions, where the driving force at the crack tip can be described with the $\Delta \mathrm{J}_{\text {eff }}$ parameter. The paper aims to give an overview of the potential applications and perspectives for the adoption of digital image correlation (DIC) measurements to study LCF nucleation and propagation. A visual technique based on the DIC displacement fields was developed to measure crack opening and closing levels in the presence of large strains. The technique is applied to different materials (steels, AlSi10Mg, nickel-based super-alloys). In addition, further applications of the DIC strain measurements are revised for the study of the nucleation stage and the crack tip process zone.
\end{abstract}

\section{Introduction}

Design approaches that consider the propagation of cracks are still currently under development as the request from industry (for example aerospace and energy) demands the application of the damage tolerance approach [1-3]. In this design perspective, a crack is supposed to nucleate from the first loading cycle in regions of the component where high stresses and strains develop [4]. Designers are then forced to consider the presence of propagating cracks in regions where the low cycle fatigue (LCF) design is considered. The overall reliability of the component is evaluated by introducing a series of safety factors, calculated following semiprobabilistic approaches, which are employed to consider the natural scatter of the external applied loads [5]. In such conditions, fatigue properties can be conservatively predicted, assuming the presence of small shallow cracks, with a depth corresponding to the detection limit of non-destructive techniques (NDT) [6].

Nowadays, advanced measurements techniques are available to characterize the mechanical behavior of metallic alloys. In particular, the digital image correlation (DIC) technique is currently under crescent interest from the engineering mechanical community $[7,8]$. The reasons for this success are different: (i) it is non-contact, (ii) it requires a (relatively) cheap set-up, (ii) it can be performed at different magnifications (providing the user can define a proper speckle pattern); (iv) it can be used to perform real-time full field measurements. DIC requires a specific specimen preparation aiming to define a target area where a proper speckle pattern has to be produced. The peculiarity of the DIC technique is that it can be used at different magnifications providing analyses that range from the macroscale (on the order of millimeters and centimeters) to the micro scale (microns).

DIC measurements are currently adopted in several applications for the mechanical characterization of materials along with the classical measurements techniques (extensometers, strain gauges, etc.). The adoption of DIC can be particularly beneficial for the characterization of the fatigue process as it allows to detect the early damage accumulation preceding the crack nucleation and, successively, to study the crack propagation stages. As an example, Abuzaid and coworkers shown that strain heterogeneities can be measured along specific microstructural features and correlated with the nucleation of cracks [9]. Displacement fields were used to calculate opening and closing levels in the high cycle fatigue (HCF) regime [10] and for calculating important fracture mechanics parameters, e.g. the stress intensity factor, [11].

In this work we discuss the application of DIC to LCF, an area where there are few applications in literature $[12,13]$. In the first part of the paper we discuss the crack closure phenomena, with particular emphasis on a technique based on DIC virtual extensometers which enables to detect the opening and closing point of a crack under LCF conditions. These results are successively validated by a second technique based on virtual strain gauges which originates from an experimental evidence presented in the work of Vormwald and co-workers, [14]. In the second part of the paper, we mainly focus on a brief discussion on the adoption of DIC strain measurements to detect early damage development during LCF loadings.

\footnotetext{
*Corresponding author: stefano.beretta@polimi.it
} 
To summarize, this study aims to provide a general overview of the potential applications of visual measurements for deepening the study of LCF behavior. Specifically, we present LCF analyses on a structural steel used in railway transportation, an AlSi10Mg alloy produced by additive manufacturing (AM) and a coarsegrained Ni-based super alloy. Prospectively, this represents a quite-large set of experimental observations which covers different industrial applications.

\section{DIC set-up and correlation strategy}

The DIC technique is based on the correlation of images of a target area captured before and during specimen deformation. The specimen surface is required to be properly prepared to produce a light-intensity pattern when observed under an optical microscope. The DIC set-up adopted in this study is composed of: (i) a 2 megapixel Allied vision Manta G201B CDD digital camera; (ii) a set of lens produced by Optem with a final magnification ranging between $10 x$ and $65 x$; (iii) a set of micro-stages to position the microscope; (iv) a Schott ACE I EKE rim light fiber optic illuminator. The final resolution of the DIC measurements performed in this study ranges between 1 and $2 \mu \mathrm{m} / \mathrm{px}$.

The specimen geometry adopted was in all cases the classical cylindrical shape with the cross-section diameter that depends on the specific material under investigation. The LCF specimens were successively machined by electro-discharge machining to obtain two symmetric flattened surfaces required to perform the DIC measurements (a schematic of the geometry is included in the inset of Fig. 1). In the case of crack propagation analysis, one or more artificial defects are machined in the flattened surfaces. For the crack nucleation tests the flattened surfaces were properly prepared on the basis of the microstructural investigation analysis required. Etching and electron-back scatter diffraction (EBSD) were used to define the grain boundary and grain orientation maps, X-ray computed tomography to detect manufacturing defects as, for example, the porosity induced by lack of fusion in AM alloys. These analyses provided an initial scan of the target surface, successively DIC measurements were correlated and overlapped to this microstructural information to correlate the specific strain heterogeneities and quantify the effect of the microstructural features.

All the experiments presented in this study were conducted under a servo-hydraulic MTS loading frame in strain control. The frequency of the cyclic loads was set to $0.5 \mathrm{~Hz}$. Depending on the specific target of the experiment, the images were manually or automatically captured. At a fixed lens magnification, the dimension of the area under investigation cannot be changed, the region of interest (ROI) is then fixed before starting the test. The test can be interrupted at a specific number of cycles or at a specific load (or deformation) and multiple images are manually captured to measure the displacement and strain fields for a large area (bigger than the single ROI). When the image acquisition is performed automatically, a video is acquired and successively un-zipped. In this case, the LCF cycle is performed at lower frequency and a number of 250 images are the generally stored for one cycle and successively correlated. For this type of acquisition, the microscope cannot be moved and the ROI is fixed to a specific specimen surface position (e.g. the crack tip).
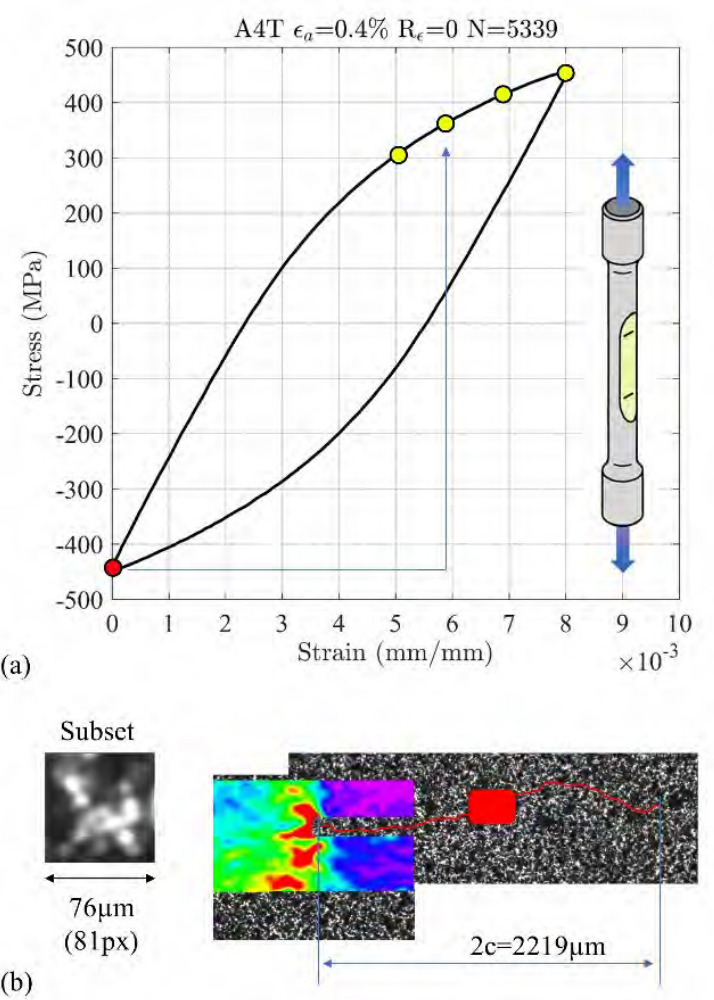

Fig. 1. DIC acquisition strategy: (a) the images are captured for the entire LCF cycle and successively correlated using the image at minimum stress as the reference one; (b) the subset size and the positioning of the DIC strain fields to locate the crack tip and calculate the crack length.

The DIC acquisition strategy for a general LCF cycle is schematically depicted in Fig. 1. The subset selected for the correlation is typically $81 \mathrm{px}$, but depending on the specific magnification required and the speckle pattern designed, smaller subsets were selected. For LCF crack propagation tests the crack tip was located using the strain fields calculated correlating the reference image taken at the minimum load (red dot in Fig. 1) with the images captured during the LCF cycle (yellow dots). The displacement and strain maps calculated with this DIC strategy refer to the incremental quantities which respect to the reference image captured in the same loading cycle. This technique was used to detect the opening and closing stresses for a LCF propagation test and the local strain ranges for a LCF nucleation test.

\section{DIC results and analyses}

In this section, the application of DIC displacement measurements is detailed for the calculation of the crack opening and closing stresses under LCF conditions. The 
most significant results are summarized for three different alloys: an A4T railway steel, an AlSi10Mg produced by SLM and a coarse-grained super-alloy. The first material is used to explain the experimental technique to calculate opening and closing levels.

\subsection{Application to A4T railway steel}

The analysis of the crack loading loop by means of DIC displacement fields is performed by the virtual extensometer technique. A sequence of virtual extensometers is positioned along the crack plane behind and after the crack tip as shown in Fig. 2a. The distance between the extensometers is $5 \mathrm{px}$ which corresponds to approximately $4.5 \mu \mathrm{m}$ considering the typical resolutions adopted for these measurements. The reading of each extensometer is plotted as a function of the applied stress in Fig. 2b. This plot provides an approximate crack profile change with loading. The crack tip is indicated at the nominal position of $d=0$.

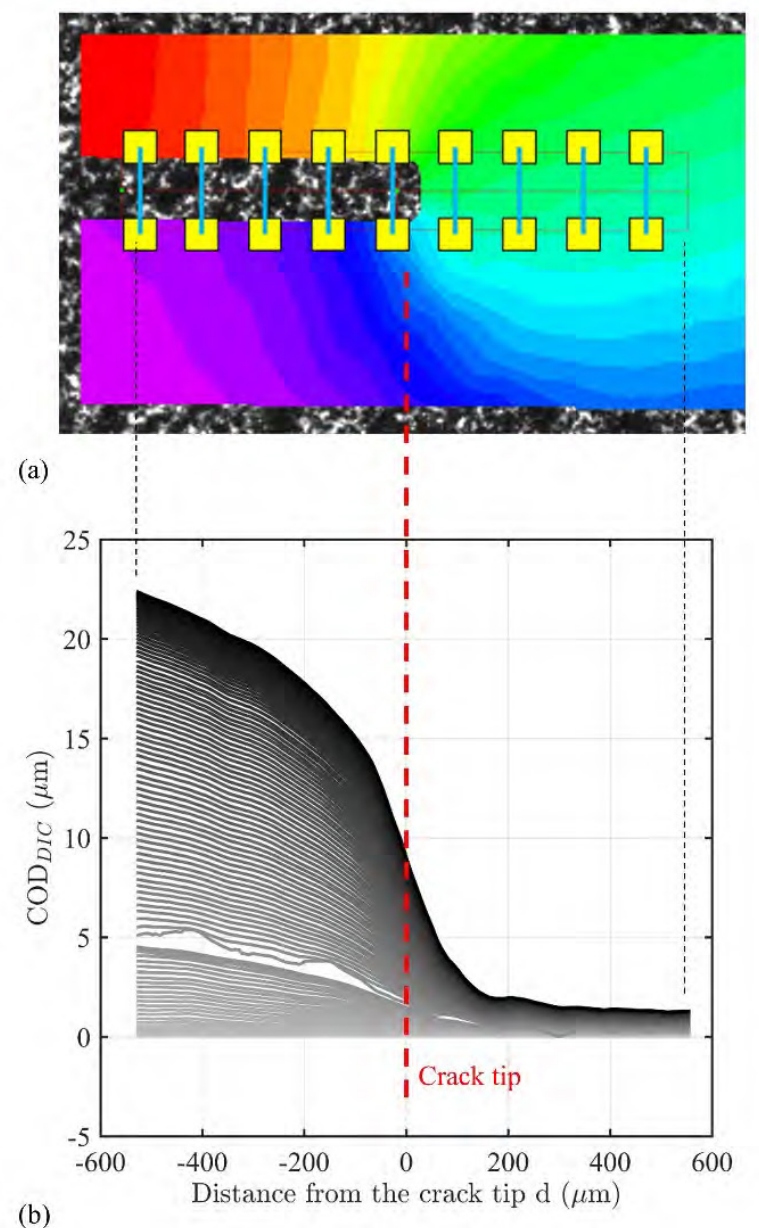

Fig. 2. Virtual extensometer readings from DIC displacement fields.

The opening stress is determined separately for each virtual extensometer reading defined as the crack opening displacement $\left(\mathrm{COD}_{\mathrm{DIC}}\right), \quad[10]$. From the minimum load is observed an initial linear relation between the remote stress and the $\mathrm{COD}_{\mathrm{DIC}}$ that originates from the linear elastic behaviour of the material in between the extensometer gauge length. In fact, the virtual extensometer cannot be positioned exactly on the crack surfaces at nominal zero distance.

After removing the initial linear part of the data set, the $\mathrm{COD}_{\mathrm{DIC}, \mathrm{pl}}$-stress plot displays an initial vertical region (Fig. 3). One possible definition of the opening is provided in [15]. The opening point is defined as the intersection between the plot with a vertical line displaced by $1.5 \%$ of the maximum $\mathrm{COD}_{\mathrm{DIC}, \mathrm{pl}}$.

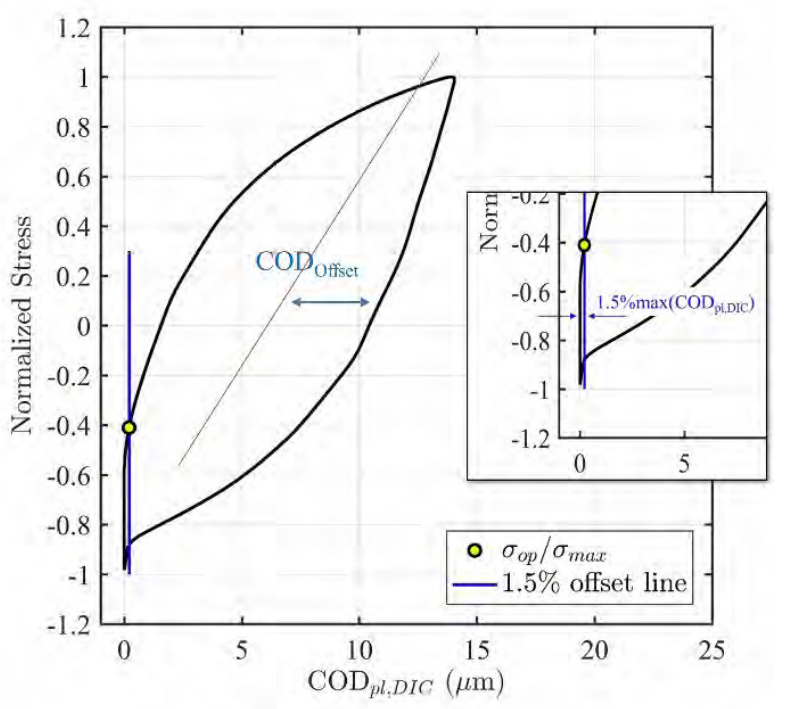

Fig. 3. The $\mathrm{COD}_{\mathrm{DIC}}$-stress plot is analysed according the $1.5 \%$ offset method to locate the opening point.

The closing point is defined according the procedure for HCF conditions suggested in [16], herein adapted for LCF. The data are linearly fitted considering the upper part of the $C O D_{D I C, p l}$-stress plot where the crack is considered to be open according an equation of the type $C O D_{D I C, p l}=m \cdot \sigma+q$. The $\mathrm{COD}_{\text {offset }}$ (Fig. 3) is then calculated for each data point according Eq. 1.

$$
C O D_{\text {offset }}=C O D_{D I C, p l}-(m \cdot \sigma+q)
$$

Plotting the $C O D_{\text {offset }}$ versus stress results in a dropshaped diagram as shown in Fig. 4a. The advantage of this representation is that it can be clearly defined the inflection point of the unloading branch which is considered the closing point of the crack tip. According to the interpretation provided in [16], the inflection point represent the point where it is observed a change in the compliance that results from unloading a plastically deformed material to the onset of crack surface contact which implies a different compliance.

An additional interpretation is also provided with the schematic drawn in Fig. 4b. During unloading, and after the crack surfaces in between the virtual extensometers get in contact, there should be observed a dominant linear relation between the $C O D_{D I C}$ and the applied load. This is observed in Fig. 3 and in Fig. 4a in correspondence of the nose of the plot. Accordingly, the closing stress precedes this nose and is consistently defined according the inflection point definition. 


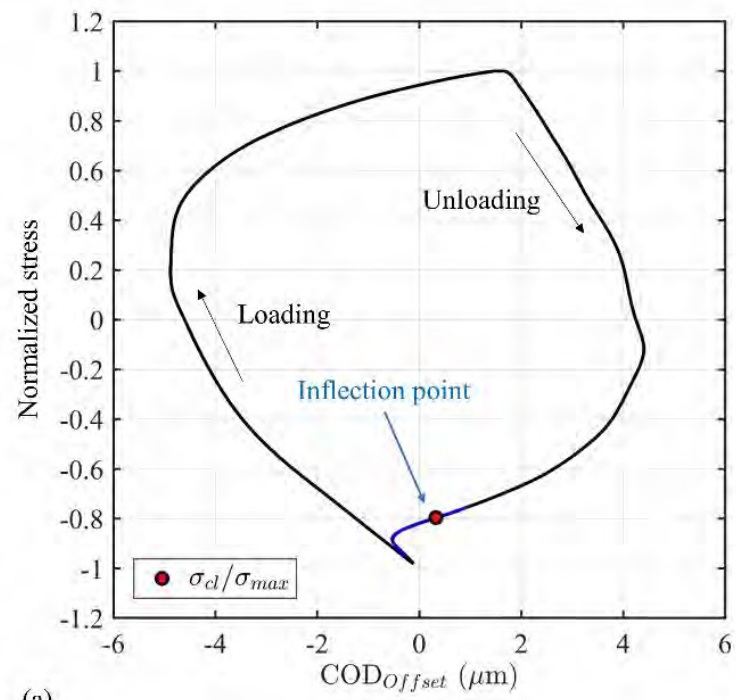

(a)
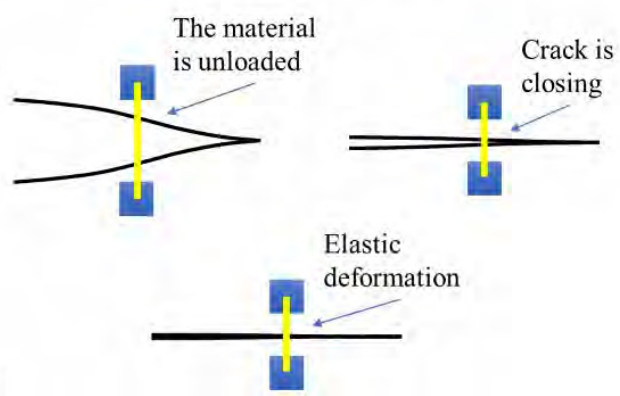

(b)

Fig. 4. Determination of the closing stress from $\mathrm{COD}_{\text {offset }}$ analysis: (a) the inflection point is determined during unloading; (b) schematic of the closing stage.

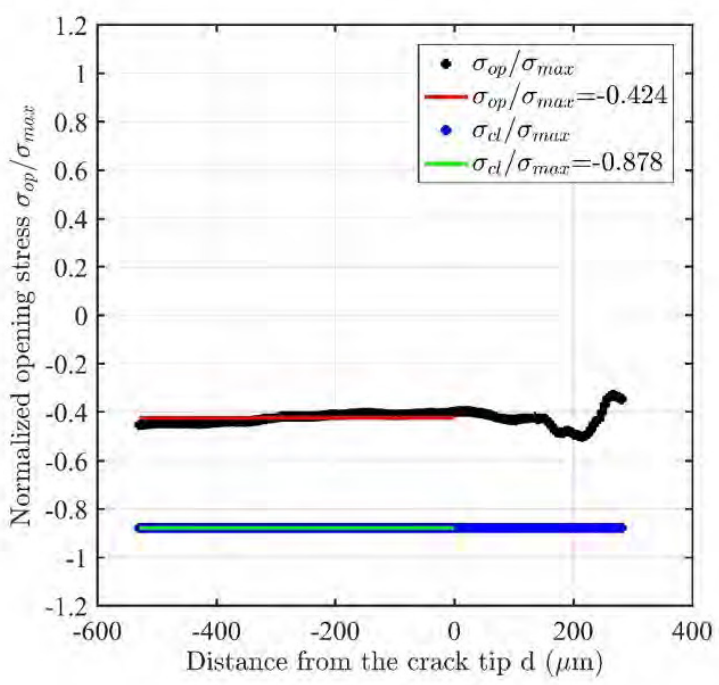

Fig. 5. Opening and closing stress measurements for different virtual extensometer position behind and ached the crack tip.

The measurements of the opening and closing stresses are then repeated for all the virtual extensometers introduced to provide a robust measurement. Fig. 5 summarizes the opening and closing levels calculation performed for the specific loading cycle and crack length. The opening and closing stresses are calculated averaging the values calculated using the virtual extensometers behind the crack tip. Noticeably, the opening stress values show a large scatter considering virtual extensometer ahead the crack tip. The number of virtual extensometers to be considered behind the crack tip is an aspect which is still under investigation. Due to the large deformations in LCF conditions, the opening stress depends on the position of the virtual extensometer. The opening stress measured decreases moving farther from the crack tip. Depending on the magnification adopted in the DIC setup, this effect has to be taken into account as it might produce over-estimation of the driving force parameter related to the opening stress level.

Following the experimental approach of Vormwald [14], local strain measurements behind the crack tip are measured by virtual strain gauges and used to detect the opening and closing stresses [13]. Fig. 8a shows one example of virtual strain gauge defined on the DIC strain fields. The blu rectangle defines the area on the DIC strain field where the local axial strain values are extracted and averaged. These strain measurements are then plotted with the bulk hysteresis cycle (Fig. 8b).

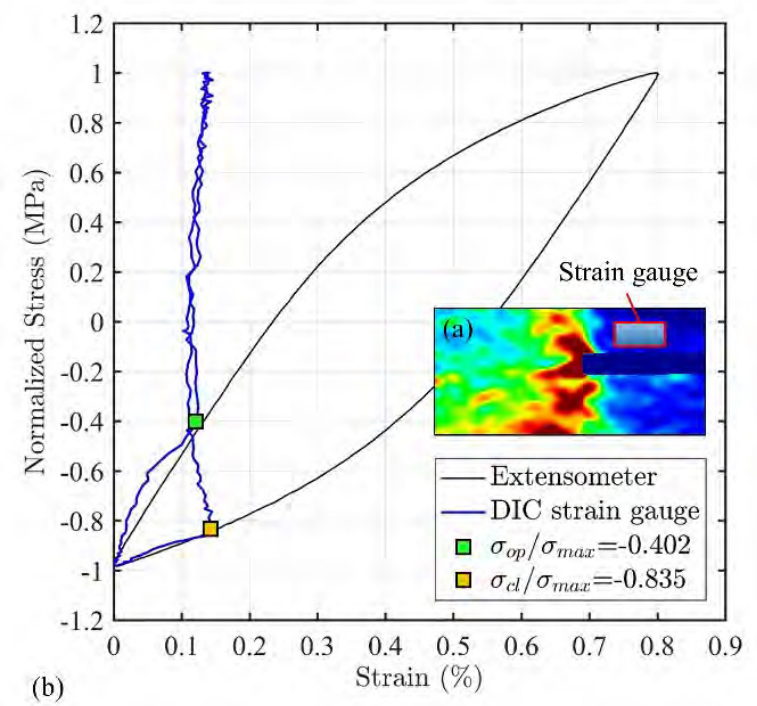

Fig. 8. Virtual strain gauge measurements for the opening and closing stresses: (a) the DIC virtual strain gauge positioned behind the crack tip; (b) the hysteresis cycle measured with the extensometer is compared with the hysteresis loop from the extensometer.

The material behaviour remains the same until the crack is closed, while after crack opening the crack surfaces in proximity of the crack tip release the elastic stress. The virtual strain gauge detects the elastic stress release displaying a vertical step in the hysteresis loop indicating that the measured strain remains constant. Once the crack surfaces are subjected to reverse loading the reading of the virtual strain gauge restores to the bulk hysteresis loop. The opening and closing levels measured with the virtual strain gauge confirms the measurements performed with the virtual extensometers (Fig. 5 and Fig. 8). 


\subsection{Application to AISi10Mg (AM)}

The aforementioned experimental technique was applied to study LCF crack propagation for an AlSi10Mg alloy produced by AM. For this material, in the current state of the manufacturing process (AM), lack of fusions and pores cannot yet be completely avoided. Design of these alloys has to deal with a crack propagation assessment [17].

The specimens were produced by means of a selective laser melting process (Fig. 6a). A semi-circular artificial defect with $200 \mu \mathrm{m}$ radius was introduced to vehiculate the crack nucleation. Three strain ranges at $R_{\varepsilon}=-1$ were selected which span from the elastic dominant behaviour $(\Delta \varepsilon=0.52 \%)$ to large plastic hysteresis loops $(\Delta \varepsilon=0.76 \%)$. The crack length was detected using DIC strain measurements as depicted in Fig. 6a. Since the axial strains are calculated correlating the images captured in one single cycle, the development of the plastic wake along the crack path is not captured within these strain maps. However, they allow to precisely locate the crack tip position and track the crack propagation path and the crack propagation rates.

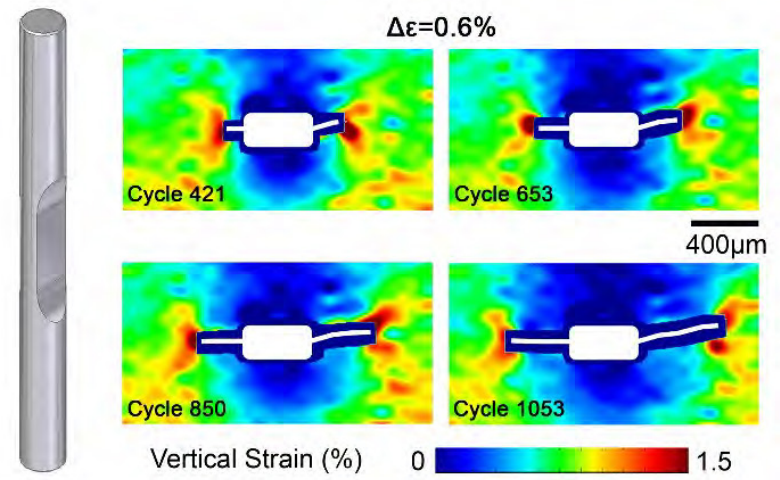

(a)

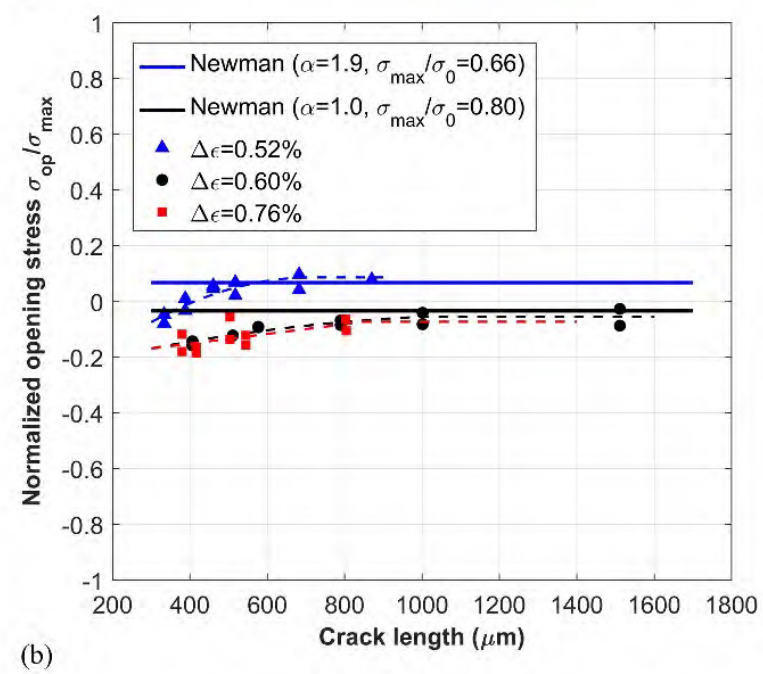

Fig. 6. Analysis of LCF crack propagation in ALSi10Mg alloy produced by AM: (a) The DIC strain fields determines the crack tip position at fixed test interruptions; (b) the normalized opening stress is shown to stabilize with crack propagation for different strain ranges [18].
The opening stresses were calculated for different crack lengths (fig.6b). The normalized opening stress was observed to increase with the number of cycles until an asymptotic value was reached. A prediction with the Newman's model [19] is also provided showing a good agreement between the stabilized opening stress levels and the ones predicted by the model.

Under fatigue loadings, local strain heterogeneities arise as a precursor of crack nucleation. In particular, microstructural features as manufacturing defects, grain boundaries, inclusions, etc can act as a local stress riser and determine the occurrence of localized plastic deformations. In the last years, a growing number of research works are based on the correlation between the detection of microstructural features and the characterization of the local strain fields that quantify the irreversible processes occurring at the micro-scale. One of the pioneering work in this field was performed on an Hastelloy X by Abuzaid and co-workers in [9]. This work demonstrated that local DIC measurements can be linked with microstructural data (grain orientations from EBSD) to quantify the specific effect of the grain boundaries in the slip transmission process. The same experimental approach was herein applied to LCF crack nucleation experiment for detecting damage development for a AlSi10Mg alloy and, in the next section, for a coarse-grained Ni-based super-alloy.
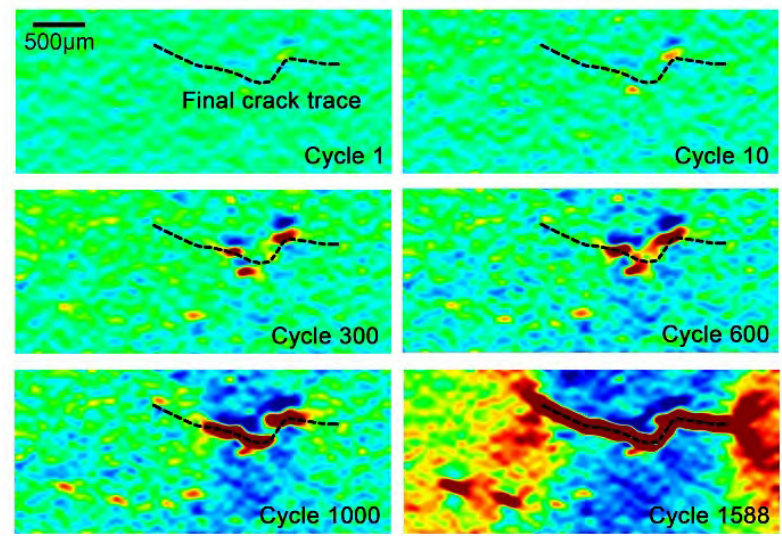

Vertical Strain (\%) -0.4

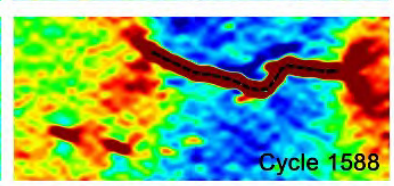

Fig. 10. Crack nucleation from pre-existing AM defects: the DIC strain localisations show the early crack nucleation from the first loading cycles in correspondence of a subsurface defect [18].

A set of cylindrical specimens for LCF tests were produced with selective laser melting process to investigate the crack nucleation for the AlSi10Mg alloy. The specimens were initially analysed by means of Xray computed tomography to detect the porosity position and size. At a fixed deformation range, in LCF conditions, there exists a critical pore dimension that determines crack nucleation. In this condition, multiple specimen locations can enhance damage initiation as multiple pores are larger than the threshold. DIC strain measurements were then performed in the area where the largest superficial defect was identified. Fig. 10 summarizes the axial strain maps measured for one 
AlSi10Mg specimen tested at $\mathrm{R}_{\varepsilon}=-1$ and $\Delta \varepsilon=0.72 \%$. The DIC measurements clearly show the early strain accumulation in a region corresponding to the specimen failure.

This study demonstrated that the fatigue life of the AlSi10Mg alloy produced by additive manufacturing can be predicted based only on a purely crack propagation model also in LCF regime as the number of cycles required to nucleate the crack is negligible compared to the specimen life. This supports the life-prediction model developed in [18].

\subsection{Application to a large-grained $\mathrm{Ni}$ - based super-alloy}

A third alloy was investigated to analyse the closure levels for more extreme microstructures. The selected alloy is a coarse-grained Ni-based super-alloy tested in LCF conditions [20]. The test temperatures were $25^{\circ} \mathrm{C}$ for the specimens $\mathrm{S} 1$ and $\mathrm{S} 2,650^{\circ} \mathrm{C}$ for the specimens S3 and S4 (Fig. 7). In these experiments, the crack path was observed to be strongly affected by the local microstructure. In addition, the elastic modulus and the yield stress depend on the specific specimen tested. Specimens S1, S2 and S4 were tested under the same strain range of $\Delta \varepsilon=0.8 \%$. The hysteresis loop shapes, so the level of plasticity, change with the local microstructure in the gauge section of the specimens. For this class of alloys, it is then mandatory to analyse and quantify the large scatter in the mechanical behaviour under the same loading conditions. In Fig. 7 the opening stress values are compared with the prediction performed with the Newman's model. As evident from the diagrams, the comparison with the Newman's model is arguable, especially for loading conditions that differ from the pure elastic material behaviour.

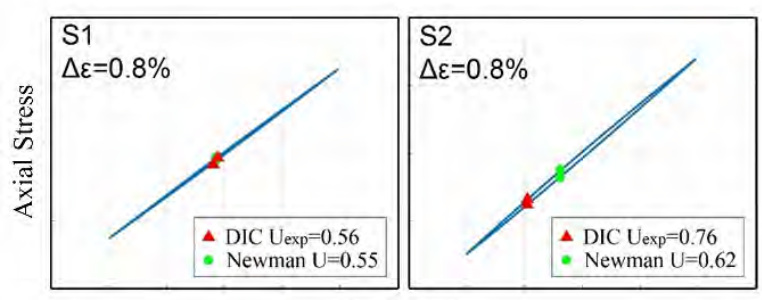

Axial Strain

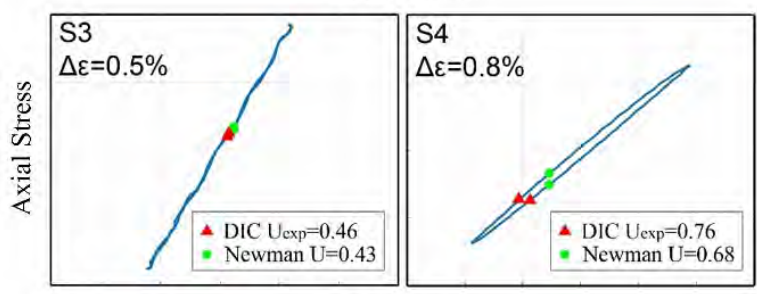

Axial Strain

Fig. 7. Opening stress measurements for a coarse-grained Nibased super-alloy [20].

High resolution DIC strain measurements were also adopted to study crack tip strain fields. Recently, experimental investigations adopting local DIC measurements were performed on a Ni-based single crystal alloy [21-23], and also on shape memory single crystals [24]. These studies highlight the importance of DIC measurements to quantify the effect of the microstructure (i.e. the crystal orientation) on the crack tip process zone. In a previous work, we reported the strain fields at the crack tip for a coarse-grained $\mathrm{Ni}$ based super-alloy [20]. Fig. 9 shows the main results of this study: the development of the crack was monitored for different specimens subjected to LCF conditions from an artificial defect. Due to the large grains, the crack tip process zone was observed to depend on the local grain orientation. The strain localisations were then used to index the slip systems which are activated depending on the grain orientation, presence of grain boundaries and orientation of the crack plane.
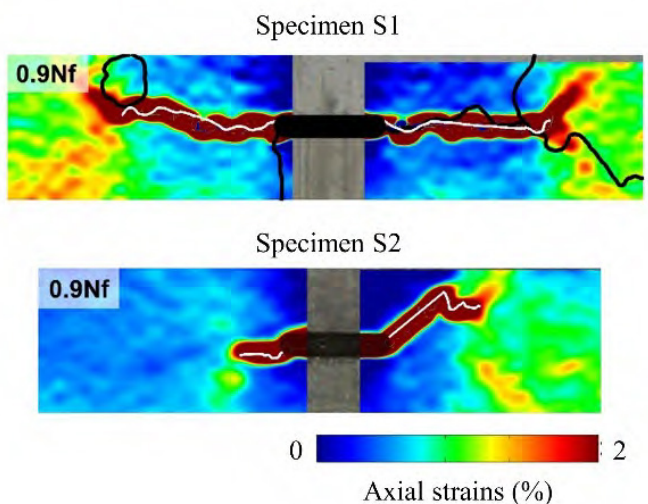

Fig. 9. Crack tip strain fields for a coarse-grained Ni-based super-alloy. The strain localisations were used to index the active slip systems [20].

For coarse-grained cast alloys, the local microstructure can promote damage initiation as a competitive mechanism with the defects originated during the manufacturing process. Specific orientations of grains contained in grain clusters can promote very high localized strains as shown, for example, in Fig. 11a.

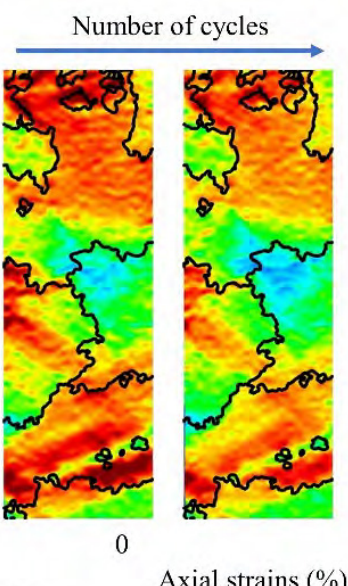

(a)
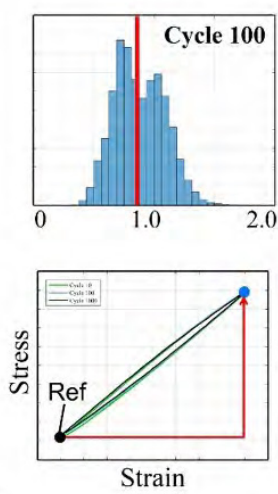

1.

(b)
Fig. 11. DIC strain measurements for a coarse-grained Nibased super-alloy: (a) high local strain heterogeneities indicate the nucleation sites and the dimension of the initial cracks; (b) correlation strategy and histogram of the strain values in the DIC area (from a private study). 
The strain maps show the axial strain along the loading direction after correlation the images captured at the minimum load with the images captured at maximum load. In this case, for the specific correlation strategy, the reported strains refer to the range displayed during one LCF cycle, and not the absolute strain accumulation during the specimen life. The values of the axial strains slightly decrease with the number of cycles. These measurements provide an important tool to evaluate the most detrimental grain boundaries which can promote crack nucleation.

\section{Final remarks}

This paper presented an overview of the potential applications of DIC measurements for material characterization under LCF conditions. The measurements were used to analyse both the nucleation and crack propagation phenomena, for different material processes (cast versus additive manufacturing) and for different grain sizes. The results support the following conclusions:

- DIC virtual extensometer technique is nowadays a robust method to detect opening and closing stress levels in LCF regime;

- Classical models for predicting crack opening and closing levels are limited for fine grainedmicrostructures, while for large grained alloys further enhancements are required;

- Crack tip strain fields improve the level of information into the crack growth mechanisms;

- High resolution DIC measurements enable to detect the heterogeneous strains which anticipate crack nucleation and quantify the local irreversibility of the damage process.

\section{References}

[1] Beretta S, Foletti S, Madia M, Cavalleri E. Structural integrity assessment of turbine discs in presence of potential defects: probabilistic analysis and implementation. Fatigue Fract Eng Mater Struct 2015;38:1042-55.

[2] Leverant GR, McClung RC, Millwater HR, Enright MP. A new tool for design and certification of aircraft turbine rotors. ASME Turbo Expo 2002 Power Land, Sea, Air, American Society of Mechanical Engineers; 2002, p. 699-704.

[3] McClung RC, Enright MP, Millwater HR, Leverant GR, Hudak SJ. A software framework for probabilistic fatigue life assessment of gas turbine engine rotors. J ASTM Int 2004;1:1-16.

[4] Murakami Y, Miller KJ. What is fatigue damage? A view point from the observation of low cycle fatigue process. Int $\mathrm{J}$ Fatigue 2005;27:991-1005.

[5] Beretta S, Foletti S, Rusconi E, Riva A, Socie D. A log-normal format for failure probability under LCF: Concept, validation and definition of design curve. Int J Fatigue 2016;82:2-11.

[6] Cristea ME, Beretta S, Altamura A. Fatigue limit assessment on seamless tubes in presence of inhomogeneities: Small crack model vs. full scale testing experiments. Int $\mathrm{J}$ Fatigue 2012;41:150-7.

[7] Chu TC, Ranson WF, Sutton MA. Applications of digital-image-correlation techniques to experimental mechanics. Exp Mech 1985;25:232-44. doi:10.1007/BF02325092.

[8] Schreier H, Orteu JJ, Sutton MA. Image Correlation for Shape, Motion and Deformation Measurements: Basic Concepts, Theory and Applications. 2009. doi:10.1007/978-0-38778747-3.

[9] Abuzaid WZ, Sangid MD, Carroll JD, Sehitoglu H, Lambros J. Slip transfer and plastic strain accumulation across grain boundaries in Hastelloy X. J Mech Phys Solids 2012;60:120120.

[10] J. Carroll, C. Efstathiou, J. Lambros, H. Sehitoglu, B. Hauber, S. Spottswood RC. Investigation of fatigue crack closure using multiscale image correlation experiments. Eng Fract Mech 2009;76:2384-2398. doi:10.1016/j.engfracmech.2009.08.002.

[11] McNeill SR, Peters WH, Sutton MA. Estimation of stress intensity factor by digital image correlation. Eng Fract Mech 1987;28:101-12.

[12] Hos Y, Freire JLF, Vormwald M. Measurements of strain fields around crack tips under proportional and non-proportional mixed-mode fatigue loading. Int J Fatigue 2015;89:87-98. doi:10.1016/j.ijfatigue.2016.01.018.

[13] Rabbolini S, Beretta S, Foletti S, Cristea ME. Crack closure effects during low cycle fatigue propagation in line pipe steel: An analysis with digital image correlation. Eng Fract Mech 2015;148:441-56. doi:10.1016/j.engfracmech.2015.07.070.

[14] Vormwald M, Seeger T. The Consequences of Short Crack Closure on Fatigue Crack Growth under Variable Amplitude Loading. Fatigue Fract Eng Mater Struct 1991;14:205-25.

[15] Pommier S, Bompard P. Bauschinger effect of alloys and plasticity-induced crack closure: a finite element analysis. Fatigue Fract Eng Mater Struct 2000;23:129-40.

[16] Chen D, Nisitani H. Analytical and experimental study of crack closure behavior based on an Sshaped unloading curve. Mech. fatigue crack Clos., ASTM International; 1988.

[17] Romano S, Brückner-Foit A, Brandão A, Gumpinger J, Ghidini T, Beretta S. Fatigue properties of AlSil0Mg obtained by additive manufacturing: Defect-based modelling and prediction of fatigue strength. Eng Fract Mech 
2017.

[18] Romano S, Patriarca L, Foletti S, Beretta S. LCF behaviour and a comprehensive life prediction model for AlSi10Mg obtained by SLM. Submitt to Int J Fatigue 2018.

[19] Newman JJC. A Crack Opening Stress Equation for Fatigue Crack Growth. Int J Fract 1984;24:R131-5.

[20] Patriarca L, Beretta S, Foletti S, Monti S, Vacchieri E. Crack closure in LCF regime for a coarse-grained Ni-based superalloy tested at high temperatures. Eighth Int. Conf. Low Cycle Fatigue, Dresden: 2017.

[21] Rabbolini S, Pataky GJ, Sehitoglu H, Beretta S. Fatigue crack growth in Haynes 230 single crystals: an analysis with digital image correlation. Fatigue Fract Eng Mater Struct 2015;38:583-96.

[22] Rabbolini S, Luccarelli PG, Beretta S, Foletti S, Sehitoglu H. Near-tip closure and cyclic plasticity in Ni-based single crystals. Int $\mathrm{J}$ Fatigue 2016;89:53-65.

[23] Beretta S, Foletti S, Rabbolini S, Sehitoglu H. Fatigue Crack Propagation in Haynes 230: A Comparison between Single and Polycrystal Crack Closure Levels. Solid State Phenom., vol. 258, Trans Tech Publ; 2017, p. 243-8.

[24] Wu Y, Ojha A, Patriarca L, Sehitoglu H. Fatigue crack growth fundamentals in shape memory alloys. Shape Mem Superelasticity 2015;1:1840. 